\title{
Trouble finding the optimal AdS/QCD
}

\author{
K. Veschgini, E. Megías*, H.J. Pirner \\ Institut für Theoretische Physik der Universität Heidelberg, Heidelberg, Germany
}

\section{A R T I C L E I N F O}

\section{Article history:}

Received 1 October 2010

Received in revised form 22 December 2010

Accepted 11 January 2011

Available online 15 January 2011

Editor: B. Grinstein

\section{Keywords:}

AdS

QCD

Holographic principle

Finite temperature

\begin{abstract}
A B S T R A C T
In the bottom-up approach to AdS/QCD based on a five-dimensional gravity dilaton action the exponential of the dilaton field is usually identified as the strong or 't Hooft coupling. There is currently no model known which fits the measurements of the running coupling and lattice results for pressure at the same time. With a one parametric toy model we demonstrate the effect of fitting the pressure on the coupling and vice versa.
\end{abstract}

(C) 2011 Elsevier B.V. All rights reserved.

\section{Introduction}

In the bottom-up approach to AdS/QCD we modify the Maldacena duality [1] between $\mathcal{N}=4$ super-Yang-Mills theory and Type IIB string theory of $\mathrm{AdS}_{5} \times S_{5}$ to find a holographic dual for QCD. We break the conformal invariance by adding a non-trivial dilaton potential $V(\phi)$ to the bulk action [2-4]

$\mathcal{S}_{\text {bulk }}=\frac{-1}{16 \pi G_{5}} \int \sqrt{G}\left(R-\frac{4}{3}\left(\partial_{\mu} \phi\right)^{2}-V(\phi)\right) \mathrm{d}^{5} x$.

The five-sphere $S_{5}$ is of no importance for the purpose of this Letter. The integration is performed over Euclidean space-time with periodic time axis and the bulk coordinate $z$. The main challenge is to find the correct potential. One approach would be to make directly an ansatz for $V$. Instead we will use $b_{0}(z)$, given by the zero temperature solution of the Einstein equations

$\mathrm{d} s^{2}=b_{0}^{2}(z)\left(\mathrm{d} \tau^{2}+\mathrm{d} \vec{x} \cdot \mathrm{d} \vec{x}+\mathrm{d} z^{2}\right)$,

to define an energy scale. The $\beta$-function is then given by

$\beta(\alpha)=b_{0} \frac{\mathrm{d} \alpha}{\mathrm{d} b_{0}}$.

The running coupling $\alpha$ on the gauge side of the duality corresponds to the exponential of the dilaton field $\exp (\phi)$. The $\beta$ -

\footnotetext{
* Corresponding author.

E-mail addresses: k.veschgini@tphys.uni-heidelberg.de (K. Veschgini), emegias@tphys.uni-heidelberg.de (E. Megías), pir@tphys.uni-heidelberg.de (H.J. Pirner).

function then fixes the potential which is obtained from the zero temperature Einstein equations $[2,3,5]$

$$
\begin{aligned}
& \partial_{z} W_{0}=\frac{16}{9} b_{0} W_{0}^{2}+\frac{3}{4} b_{0} V\left(\alpha_{0}\right), \\
& \partial_{z} b_{0}=-\frac{4}{9} b_{0}^{2} W_{0}, \\
& \partial_{z} \alpha_{0}=\alpha_{0} \sqrt{b_{0} \partial_{z} W_{0}} . \\
& \text { namely }^{1}
\end{aligned}
$$$$
V(\alpha)=-\frac{12}{\ell^{2}} \exp \left(-\frac{8}{9} \int_{0}^{\alpha} \frac{\beta(\tilde{\alpha})}{\tilde{\alpha}^{2}} \mathrm{~d} \tilde{\alpha}\right)\left(1-\left(\frac{\beta(\alpha)}{3 \alpha}\right)^{2}\right) .
$$

$W_{0}$ is defined by Eq. (1.5) to reduce Einstein equations to first order. Thus, up to the constant factor $12 / \ell^{2}$ the dilaton potential is fixed by the $\beta$-function.

A holographic model is meant to capture infrared physics as, according to the AdS/CFT correspondence the gravity description of the super-Yang-Mills theory applies to the large 't Hooft coupling limit $\lambda_{t}=g_{\mathrm{YM}}^{2} N_{c} \rightarrow \infty$. The ultraviolet behavior physics computed from a gravity dual is known to show often a wrong behavior. For example, consider a holographic model with the $\beta$-function

$\beta_{\text {pert }}(\alpha)=-\beta_{0} \alpha^{2}-\beta_{1} \alpha^{3}$,

in the ultraviolet. The asymptotic behavior of the spatial string tension computed from the gravity dual in the limit $T \rightarrow \infty$ is

\footnotetext{
1 The minus signs in the dilaton potential and in front of $V(\phi)$ in Eq. (1.1) are a matter of convention.
} 
$\sigma_{s} \propto T^{2} \alpha^{4 / 3}[6,7]$ instead of $\sigma_{s} \propto T^{2} \alpha^{2}$ as it follows from dimensional reduction arguments and lattice simulations [8,9]. Similarly if we compare the asymptotic behavior of the pressure [10,7]

$$
\begin{aligned}
p & =\frac{\pi^{3} \ell^{3}}{16 G_{5}} T^{4}\left(1-\frac{4}{3} \beta_{0} \alpha_{h}+\frac{2}{9}\left(4 \beta_{0}^{2}-3 \beta_{1}\right) \alpha_{h}^{2}\right) \\
& =p_{\mathrm{SB}}\left(1-2.33 \alpha_{h}+1.86 \alpha_{h}^{2}\right),
\end{aligned}
$$

with the perturbative result from QCD [11]

$$
\begin{aligned}
p & =\frac{8 \pi^{2}}{45} T^{4}\left(1-\frac{15}{4} \frac{\alpha}{\pi}+30\left(\frac{\alpha}{\pi}\right)^{3 / 2}\right) \\
& =p_{\mathrm{SB}}\left(1-1.19 \alpha+5.4 \alpha^{3 / 2}\right),
\end{aligned}
$$

we see that not only the coefficients are different but also the power of $\alpha$ in the next to leading order. Note, perturbation theory gives the coupling at the black hole horizon $\alpha_{h}=\alpha\left(z_{h}\right)$ equal to $\alpha(\pi T)$ in QCD in lowest order. In many cases, like the spatial string tension, the ultraviolet limit does not prevent the model from capturing the infrared physics, but in the case of the pressure the situation is different. The gravity model suggests a smaller pressure than perturbative QCD at very high temperatures, as we can see from a comparison of the coefficients at $\mathcal{O}(\alpha)$ in Eqs. (1.9) and (1.10). If the pressure is already much too small at $10^{3} T_{c}$, it has a tendency to be also much too small at lower temperatures.

We will consider a simple $\beta$-function and demonstrate that as we switch over at smaller values of $\hat{\alpha}$ from $\beta_{\text {pert }}$ to an asymptotic linear behavior the pressure gets larger. Fitting lattice results requires $\hat{\alpha} \lesssim 0.04$.

\section{The model}

We assume the following toy $\beta$-function to demonstrate our case:

$\beta(\alpha)= \begin{cases}\beta_{\text {pert }}(\alpha)-\hat{\beta}_{2} \alpha^{4} & \text { if } \alpha \leqslant \hat{\alpha}, \\ \beta_{\text {pert }}(\hat{\alpha})-\hat{\beta}_{2} \hat{\alpha}^{4}-3(\alpha-\hat{\alpha}) & \text { if } \alpha>\hat{\alpha},\end{cases}$

where

$\hat{\beta}_{2}=\frac{3-2 \beta_{0} \hat{\alpha}-3 \beta_{1} \hat{\alpha}^{2}}{4 \hat{\alpha}^{3}}$,

is chosen such that $\partial_{\alpha} \beta(\alpha)$ is continuous. There is only one parameter $\hat{\alpha}$ which controls the transition point. Note that $\hat{\beta}_{2}$ will become very large for small $\hat{\alpha}$ such that $\beta(\alpha)$ deviates from $\beta_{\text {pert }}(\alpha)$ already at $\alpha$ much smaller than $\hat{\alpha}$. The slope of the linear term is chosen to be -3 . With this choice, according to Eq. (1.7) we obtain a monotonic potential with the following asymptotic behaviors:

$$
\begin{aligned}
& V(\alpha) \rightarrow-12 / \ell^{2} ; \quad \alpha \rightarrow 0, \\
& V(\alpha) \sim-\alpha^{5 / 3} ; \quad \alpha \rightarrow \infty .
\end{aligned}
$$

\section{The pressure}

The deconfined phase of the gluon plasma is described in the holographic picture by a black hole geometry with the metric

$\mathrm{d} s^{2}=b^{2}(z)\left(f(z) \mathrm{d} \tau^{2}+\mathrm{d} \vec{x} \cdot \mathrm{d} \vec{x}+\frac{\mathrm{d} z^{2}}{f(z)}\right)$.

The horizon lies in the bulk at $z_{h}$ where $f\left(z_{h}\right)=0$. We normalize $f(0)$ to 1 . In order to avoid a conical singularity the periodicity of the $\tau$ axes $\beta=T^{-1}$ must be fixed to [12,13]

$\beta=-\left.\frac{4 \pi}{\partial_{z} f(z)}\right|_{z=z_{h}}$.

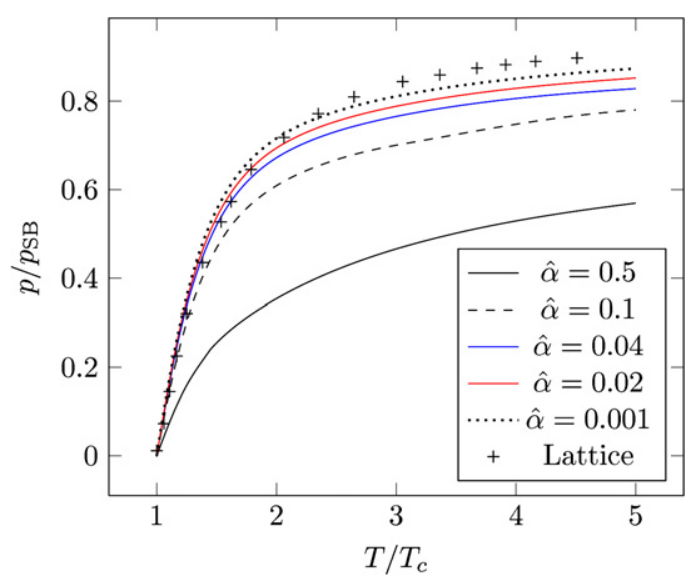

Fig. 1. Pressure normalized to the Stefan-Boltzmann pressure as a function of $T / T_{C}$ compared with lattice data for $N_{c}=3$ taken from Ref. [8].

The zero temperature solution serves as background. Any finite temperature solution shares the same ultraviolet behavior as the zero temperature solution up to $\mathcal{O}\left(z^{4}\right)$. The physical action is given by the difference between the action of the black hole solutions and the zero temperature solution

$\mathcal{S}_{\text {phys. }}=\mathcal{S}_{T}-\mathcal{S}_{0}$,

where $\mathcal{S}_{T}$ and $\mathcal{S}_{0}$ are actions given by the bulk term Eq. (1.1) plus the Gibbons-Hawking-York boundary term [13,14]

$\mathcal{S}_{\text {bound. }}=\frac{1}{8 \pi G_{5}} \int \mathrm{d} x^{4} \sqrt{\mathrm{g}} K$.

The induced metric on the boundary is $g$ and $K$ is the trace of the second fundamental form of the boundary. For temperatures $T$ larger than some $T_{\min }$ we find three solutions, the zero temperature solution (temperature independent) and two black hole solutions. For $T<T_{\min }$ the black hole solutions are not present. The solution with the smaller physical action defines the stable gluonic matter. The phase of confined gluons is given from $T=0$ up to $T=T_{c}$ by the zero temperature solution. Black hole solutions exist for temperatures higher than some $T_{\min }$, the big black holes have a smaller action than the small black holes and for $T>T_{c}$ a negative physical action (relative to the $T=0$ solution). The black hole geometry corresponds to the deconfined phase. Details of the computation can be found in Ref. [7]. The free energy can be computed directly from the physical action as $\mathcal{F}=T \mathcal{S}_{\text {phys. }}$ or by integrating the entropy

$\mathcal{F}=\int S \mathrm{~d} T$.

The entropy of a classical black hole is a well defined quantity given by the Bekenstein-Hawking formula $[15,16]$

$S=\frac{A}{4 G_{5}}=\frac{\operatorname{Vol}(3) b^{3}\left(z_{h}\right)}{4 G_{5}}$,

where $A$ is the area of the black hole horizon. Both methods produce the same result but the latter approach is numerically favorable [7]. Thermodynamic quantities can then be computed from the free energy. The five-dimensional gravitational constant $G_{5}$ is fixed by normalizing the pressure given in Eq. (1.9) to the StefanBoltzmann pressure in the limit $T \rightarrow \infty$ :

$G_{5}=\frac{45 \pi \ell^{3}}{16\left(N_{c}^{2}-1\right)}$.

This value differs from the conformal case because we have fewer degrees of freedom in QCD than in $\mathcal{N}=4$ super Yang-Mills 


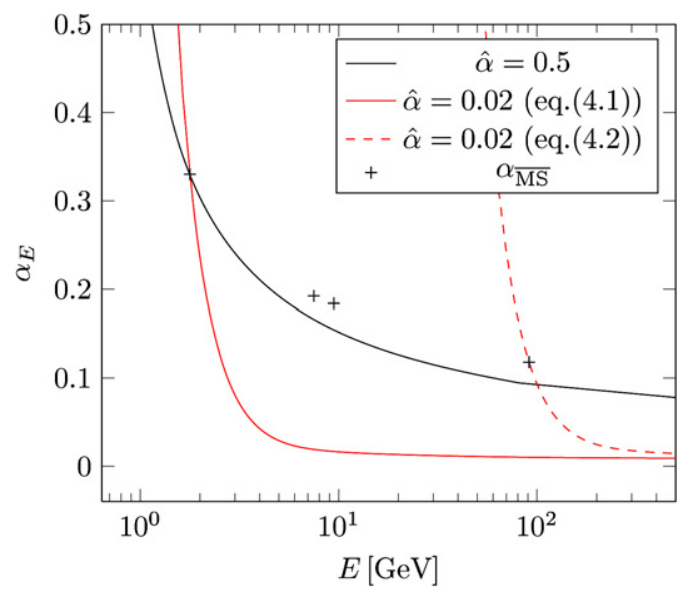

Fig. 2. The running coupling $\alpha$ as a function energy $E=\Lambda_{E} b_{0}$. Data points are taken from Ref. [18]. The curve corresponding to $\hat{\alpha}=0.02$ is shown for two different choices of $\Lambda_{E}$

theory [17]. We have computed the pressure using BekensteinHawking entropy for different choices of $\hat{\alpha}$. The results normalized to the ideal gas limit are shown in Fig. 1. Lattice data from $T_{c}$ up to $2 T_{c}$ are fitted very well for $\hat{\alpha}=0.04$. The choice $\hat{\alpha}=0.02$ gives the best fit from $T_{c}$ up to $3 T_{c}$. Some other curves are also shown for comparison. Data above $3 T_{c}$ are not fitted precisely by any choice of $\hat{\alpha}$.

\section{The running coupling and screening mass}

It is interesting to see how the running coupling $\alpha$ is affected by the choice of $\hat{\alpha}$. The weak coupling expansion Eq. (1.9) already suggests that the coupling must be very small when we want to fit the pressure. First we discuss the zero temperature running coupling as a function of energy $E$, which we denote by $\alpha_{E}$. Since we define the $\beta$-function with $b_{0}$ as given in Eq. (1.3), the energy is proportional to $b_{0}(z)$. The proportionality constant $\Lambda_{E}$ is not fixed by the model. Different values of $\Lambda_{E}$ correspond to different initial conditions for the renormalization group equation (1.3). Fig. 2 shows $\alpha_{E}$ as a function of energy $E$ in logarithmic scale. Different values of $\Lambda_{E}$ shift the curves to the left or right without affecting their shape since $\log \left(E=\Lambda_{E} b_{0}\right)=\log \left(\Lambda_{E}\right)+\log \left(b_{0}\right)$. This is demonstrated for the case $\hat{\alpha}=0.02$, where we fix $\Lambda_{E}$ either by

$\alpha_{E}(1.78 \mathrm{GeV})=0.33$

or by

$\alpha_{E}\left(M_{Z}=91.2 \mathrm{GeV}\right)=0.118$

according to Ref. [18]. A small value of $\hat{\alpha}$ results in a very small coupling at high energies. As a side effect the curve becomes also very flat, there is almost no running at high energies. On the other hand the coupling rises extremely fast in the infrared. This differs from the case $\hat{\alpha}=0.5$ which is a much better fit to the $\overline{\mathrm{MS}}$ value in the plotted range.

The chosen $\beta$-function for $\hat{\alpha}=0.5$ can reproduce the running coupling in vacuum quite well. The coupling at finite temperatures defined as $\alpha_{h}(T)=\alpha\left(z_{h}\right)$ follows the vacuum coupling constant in the ultraviolet [4,7]. Thus $\alpha_{h}$ is also very small at high temperatures. In order to test the model at finite temperatures $T$, it is important to monitor the behavior of an observable which is closely related to the coupling at finite temperatures. To this end we have computed the Debye mass in the gluon plasma. We use

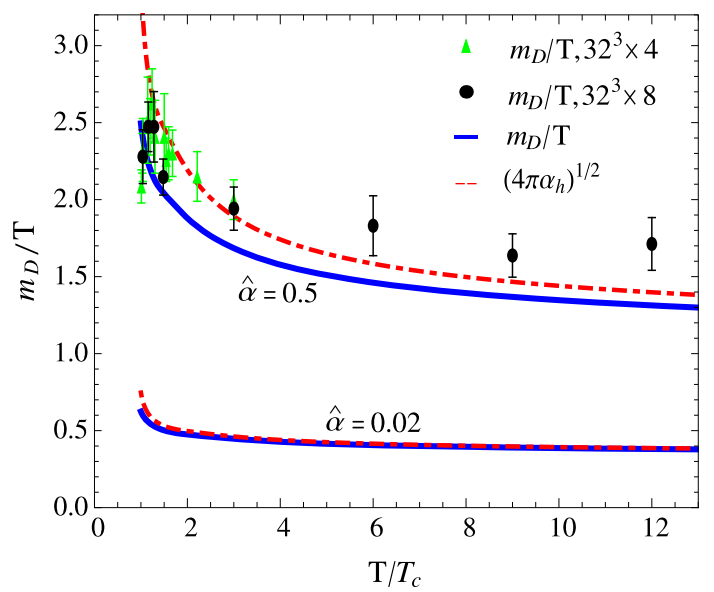

Fig. 3. Debye mass over temperature as a function of $T / T_{c}$. The full (blue) curves correspond to computations using $\hat{\alpha}=0.02$ and $\hat{\alpha}=0.5$. Lattice data are taken for gluodynamics SU(3) with $N_{\sigma}^{3} \times N_{\tau}=32^{3} \times 4$ and $32^{3} \times 8$ from Refs. [20,21]. The dot dashed (red) curves represent the finite temperature running coupling $\left(4 \pi \alpha\left(z_{h}\right)\right)^{1 / 2}$ for both cases. (For interpretation of the references to colour in this figure legend, the reader is referred to the web version of this Letter.)

the method presented in Ref. [19]. The results for $\hat{\alpha}=0.02$ and $\hat{\alpha}=0.5$ are plotted in Fig. 3 together with lattice data $[20,21]$. The model with $\hat{\alpha}=0.02$ predicts a value for the Debye mass which is a factor 4 smaller than lattice data. This is a consequence of the fact that the running coupling $\alpha_{h}$ is very small at high temperature for this choice of $\hat{\alpha}$. For the large $\hat{\alpha}=0.5$ the Debye mass is quite close to the lattice data as one can see in Fig. 3.

\section{Summary and conclusion}

The $\beta$-function, on the one hand defines the running of the coupling and on the other hand determines the dilaton potential and hence the full thermodynamics including the pressure and the screening mass. Following the idea of AdS/CFT we identify the exponential of the dilaton field as the running coupling of QCD. But, when we fit the pressure, the running coupling $\alpha$ and the screening mass in the plasma $m_{D}$ deviate strongly from phenomenology. On the other side, if we choose to fit $\alpha$ or $m_{D}$, we obtain a pressure that is about $40 \%$ too small. This outcome is not just a property of the model introduced here cf. $[22,10,23]$. In the literature Refs. $[22,10]$ both approaches have been covered. In ref. [22] a very good description of the thermodynamics has been achieved, however at the expense of not being able to relate the dilaton potential with the running $\alpha(E)$ in the $\overline{\mathrm{MS}}$ scheme. Note, one could argue, that in the scheme used here with a large coefficient $\hat{\beta}_{2} \alpha^{4}$ in the $\beta$-function, the $\overline{\mathrm{MS}}$-value of $\alpha_{\overline{\mathrm{MS}}}(E)=0.33$ at $E=1.78 \mathrm{GeV}$ is not realistic. But as shown in Section 4 any other choice leads to the same result. Also the second thermodynamic observable, the Debye mass, points to a real deficit for this choice of small $\hat{\alpha}$. For our toy model as well as for the model of Ref. [22] there is currently no known mapping between the assumed $\beta$-function and known $\overline{\mathrm{MS}}$ values of $\alpha$ [24]. This would be very much needed if one wants to apply this model to hadronization. In Ref. [10] an extrapolation of the $\beta$-function was used which agrees well the perturbative running of $\alpha_{\overline{\mathrm{MS}}}$ [5]. The calculations of the thermodynamics however have the default shown by the toy-model for $\hat{\alpha} \approx 0.5$. It produces a pressure which is at all temperatures too small, whereas the calculation of the Debye mass comes out in agreement with the lattice data. At the moment we do not know any solution of this dilemma. 


\section{Acknowledgements}

E.M. would like to thank the Humboldt Foundation for their stipend. This work was also supported in part by the ExtreMe Matter Institute EMMI in the framework of the Helmholtz Alliance Program of the Helmholtz Association.

\section{References}

[1] J.M. Maldacena, Adv. Theor. Math. Phys. 2 (1998) 231, arXiv:hep-th/9711200

[2] U. Gursoy, E. Kiritsis, J. High Energy Phys. 0802 (2008) 032, arXiv:0707.1324 [hep-th].

[3] U. Gursoy, E. Kiritsis, F. Nitti, J. High Energy Phys. 0802 (2008) 019, arXiv: 0707.1349 [hep-th].

[4] U. Gursoy, E. Kiritsis, L. Mazzanti, F. Nitti, J. High Energy Phys. 0905 (2009) 033. arXiv:0812.0792 [hep-th].

[5] B. Galow, E. Megias, J. Nian, H.J. Pirner, Nuclear Phys. B 834 (2010) 330, arXiv: 0911.0627 [hep-phj].

[6] J. Alanen, K. Kajantie, V. Suur-Uski, Phys. Rev. D 80 (2009) 075017, arXiv: 0905.2032 [hep-ph].

[7] E. Megias, H.J. Pirner, K. Veschgini, 2010, arXiv:1009.2953 [hep-ph].

[8] G. Boyd, et al., Nuclear Phys. B 469 (1996) 419, arXiv:hep-lat/9602007.
[9] B. Lucini, M. Teper, Phys. Rev. D 66 (2002) 097502, arXiv:hep-lat/0206027.

[10] E. Megias, H.J. Pirner, K. Veschgini, Nucl. Phys. B (Proc. Suppl.) 207-208 (2010) 333, arXiv:1008.4505 [hep-th].

[11] K. Kajantie, M. Laine, K. Rummukainen, Y. Schroder, Phys. Rev. D 67 (2003) 105008, arXiv:hep-ph/0211321.

[12] S. Carlip, Lecture Notes in Phys. 769 (2009) 89, arXiv:0807.4520 [gr-qc].

[13] G.W. Gibbons, S.W. Hawking, Phys. Rev. D 15 (May 1977) 2752.

[14] J.W. York, Phys. Rev. Lett. 28 (Apr 1972) 1082.

[15] J.D. Bekenstein, Phys. Rev. D 7 (1973) 2333.

[16] S.W. Hawking, Comm. Math. Phys. 43 (1975) 199.

[17] T. Schafer, D. Teaney, Rept. Prog. Phys. 72 (2009) 126001, arXiv:0904.3107 [hep-ph].

[18] S. Bethke, Eur. Phys. J. C 64 (2009) 689, arXiv:0908.1135 [hep-ph].

[19] A. Gorsky, P.N. Kopnin, A. Krikun, 2010, arXiv:1012.1478 [hep-ph].

[20] O. Kaczmarek, F. Karsch, F. Zantow, P. Petreczky, Phys. Rev. D 70 (2004) 074505 arXiv:hep-lat/0406036.

[21] O. Kaczmarek, F. Zantow, Phys. Rev. D 71 (2005) 114510, arXiv:hep-lat/ 0503017.

[22] U. Gursoy, E. Kiritsis, L. Mazzanti, F. Nitti, Nuclear Phys. B 820 (2009) 148 arXiv:0903.2859 [hep-th].

[23] J. Alanen, K. Kajantie, V. Suur-Uski, Phys. Rev. D 80 (2009) 126008, arXiv: 0911.2114 [hep-ph].

[24] U. Gursoy, E. Kiritsis, L. Mazzanti, G. Michalogiorgakis, F. Nitti, 2010, arXiv: 1006.5461 [hep-th]. 